\title{
Analysis of the association of MIRI24-I and its target gene RGS4 polymorphisms with major depressive disorder and antidepressant response
}

This article was published in the following Dove Press journal:

Neuropsychiatric Disease and Treatment

\author{
Duan Zengl,* \\ Shen $\mathrm{He}^{1, *}$ \\ Shunying $\mathrm{Yu}^{\prime}$ \\ Guanjun $\mathrm{Li}^{\prime}$ \\ Changlin $\mathrm{Ma}^{2}$ \\ YiWen ${ }^{2}$ \\ Yifeng Shen' \\ Yimin $\mathrm{Yu}^{\prime}$ \\ Huafang $\mathrm{Li}^{1,3}$ \\ 'Department of Psychiatry, Shanghai \\ Mental Health Center, Shanghai \\ Jiao Tong University School of \\ Medicine, Shanghai, People's \\ Republic of China; ${ }^{2}$ Shanghai Jiading \\ District Mental Health Center, \\ Shanghai, People's Republic of China; \\ ${ }^{3}$ Shanghai Key Laboratory of Psychotic \\ Disorders, Shanghai Mental Health \\ Center, Shanghai Jiao Tong University \\ School of Medicine, Shanghai, \\ People's Republic of China \\ *These authors contributed equally \\ to this work
}

Background: Increasing evidence has indicated that dysfunction of miR-124 and target gene regulator of G protein signaling 4 (RGS4) may be involved in the etiology and treatment of major depressive disorder (MDD). However, the molecular mechanisms are not fully understood. This study aimed to investigate whether common genetic variations in these two genes are associated with MDD and therapeutic response to antidepressants in the Chinese population.

Methods: Three polymorphisms including rs531564 (a functional single-nucleotide polymorphism [SNP] in MIR124-1), rs10759 (a microRNA-binding site SNP in RGS4), and rs951436 (a promoter SNP in RGS4) were genotyped in 225 Chinese MDD patients and 436 controls. Among the MDD patients, 147 accepted antidepressant treatment for 8 weeks with therapeutic evaluation at baseline, week 2, week 4, week 6, and week 8 using the 17-item Hamilton Rating Scale for Depression. Multifactor dimensionality reduction (MDR) was used to identify genegene interactions.

Results: No significant association with MDD was discovered in single-SNP analyses. However, by MDR analysis, the three-locus model of gene-gene interaction was the best for predicting MDD risk. In pharmacogenetic study, a significant association was found in genotypic frequencies of rs 951436 between the remitter and non-remitter groups ( $p=0.026$, correction $p=0.078$ ). For further analysis, the rs 951436 heterozygote carriers had threefold probabilities of achieving clinical complete remission (odds ratio $=3.00,95 \%$ confidence interval $=1.33-6.76, p=0.007$, correction $p=0.021$ ) as compared with rs951436 homozygotes (AA+CC) after 8 weeks of treatment.

Conclusion: An interaction effect of MIR124-1 and RGS4 polymorphisms may play a more important role than individual factors for MDD development. Moreover, RGS4 gene polymorphisms may be associated with antidepressant response among the Han population.

Keywords: polymorphisms, MIR124-1, regulator of G protein signaling 4, depression, antidepressant, gene-gene interaction

\section{Introduction}

Major depressive disorder (MDD) is a common psychiatric disorder and a leading cause of disability worldwide. However, the etiology of MDD and the mechanisms of antidepressant treatment have not been clearly elucidated.

MicroRNAs (miRNAs) are small noncoding single-stranded RNAs, which are believed to regulate the expression of target mRNA by binding to sites of antisense complementarity in $3^{\prime}$ untranslated regions ( $3^{\prime}$ UTRs). ${ }^{1}$ miRNAs play a fundamental role in the development of brain and influence neuronal and circuit formation by negatively regulating gene expression. ${ }^{2}$ Increasing evidence has shown that the dysfunction of miRNAs and their targets may be critically involved in MDD and related disorders. ${ }^{2,3}$
Correspondence: Huafang Li; Yifeng Shen Shanghai Mental Health Center, Shanghai Jiao Tong University School of Medicine, 600 South Wan ping Road, Shanghai, 200030, People's Republic of China Tel +86 2I 34773657 Email lhlh_5@I63.com; shenyifeng@yahoo.com 
Among miRNAs, miR-124 is the most abundant miRNA in the adult brain and plays an important role in neuronal differentiation, neurogenesis, and neuronal regeneration. ${ }^{4} \mathrm{miR}-124$ regulates key genes for MDD such as regulator of $\mathrm{G}$ protein signaling 4 (RGS4), SRY-box 9 (SOX9), nuclear receptor subfamily 3 group C member 1 (NR3C1), AKT1 substrate 1 (AKT1S1), glutamate ionotropic receptor AMPA-type subunit 3 and subunit 4 (GRIA3 and GRIA4). ${ }^{5-7}$ It also regulates key pathways, such as brain-derived neurotrophic factor-TrkB, glucocorticoid receptor, and Jagged-1-Notch signaling pathways. ${ }^{3,8,9}$ These genes and pathways could be involved in the top-down and bottom-up pathophysiological mechanisms of MDD.

Emerging evidence has demonstrated that dysregulation of miR-124 expression occurs in rodent depression models, ${ }^{10}$ in the postmortem brain tissue of depressed patients, and in the serum of depressed subjects. ${ }^{5}$ Bahi et $\mathrm{al}^{3}$ found that hippocampal miR-124 overexpression exacerbated stress-induced depression-like behavior. Moreover, an antidepressant-like effect, such as less immobile and more active in swimming behavior, was observed when miR124a silencers were injected into the hippocampus. ${ }^{3}$ Furthermore, our recently published data demonstrated that expression levels of miR-124 in peripheral blood mononuclear cells of patients were significantly higher than those in controls and significantly downregulated after 8 weeks of treatment. ${ }^{11}$ Therefore, we speculate that miR-124 may be associated with MDD pathogenesis and antidepressant efficacy.

Three miRNA genes including MIR124-1 (8p23.1), MIR124-2 (8q12.3), and MIR124-3 (20q13.33) encode miR124 in the human genome. A functional single-nucleotide polymorphism (SNP) rs531564 (g.9.903.189C $>$ G) located in the pri-miRNA region of the MIR124-1 gene led to the different expression levels of the mature miR-124. ${ }^{12}$ Several studies have evaluated the association of rs531564 with Alzheimer's disease and aggressiveness. ${ }^{12,13}$ However, whether rs531564 is associated with MDD susceptibility and antidepressant treatment is unclear.

$R G S 4$, one of the validated target genes of miR-124, is widely distributed and highly expressed in many brain regions, including the cerebral cortex, striatum, thalamus, and brainstem nuclei. ${ }^{14} R G S 4$ is known to regulate postreceptor signaling cascades for vast G-protein-coupled receptors (GPCRs), including serotonin, adrenergic, dopamine, muscarinic, and opioid receptors. Guilloux et al found that $R G S 4$ gene expression was downregulated in the amygdala of postmortem MDD subjects. ${ }^{15}$ Stratinaki et al found robust upregulation of $R G S 4$ expression in postmortem brain tissue of MDD patients receiving standard antidepressant medications that target monoamine systems, and similar results were found in mouse models. ${ }^{16}$ Therefore, we speculate that RGS4 is involved in the pathology of MDD and a potential target for antidepressant treatments.

Polymorphisms in 3' UTRs of genes may affect miRNA binding to specific mRNAs and contribute to the risk of diseases. ${ }^{7}$ rs10759 polymorphism, located in the 3' UTRs of RGS4 gene, was found to increase the risk of schizophrenia by altering the binding of miRNA-124 to its target (Figure 1). ${ }^{7}$ In addition, Buckholtz et al found that rs951436, another polymorphism of the RGS4 gene, was associated with regionally specific reductions in the structural volume of gray and white matter, which is involved in mood, movement, and cognition. ${ }^{17}$ Therefore, we questioned whether the two SNPs of RGS4 were associated with MDD and antidepressant efficacy in the Chinese population.

The aim of the present study was to investigate the association of the functional SNP rs531564 of the MIR124-1 gene and the two SNPs (rs10759 and rs951436) of its target gene RGS4 with MDD pathology and antidepressant treatment.

\section{Methods \\ Participants}

The study population consisted of 225 outpatients from Shanghai Mental Health Center, China. All subjects were assessed by trained psychiatrists using the Structured Clinical Interview for Diagnostic and Statistical Manual of Mental Disorders, Fourth Edition (DSM-IV) Axis-I Disorders. The inclusion criteria were as follows: 1) Han ethnicity, 2) age

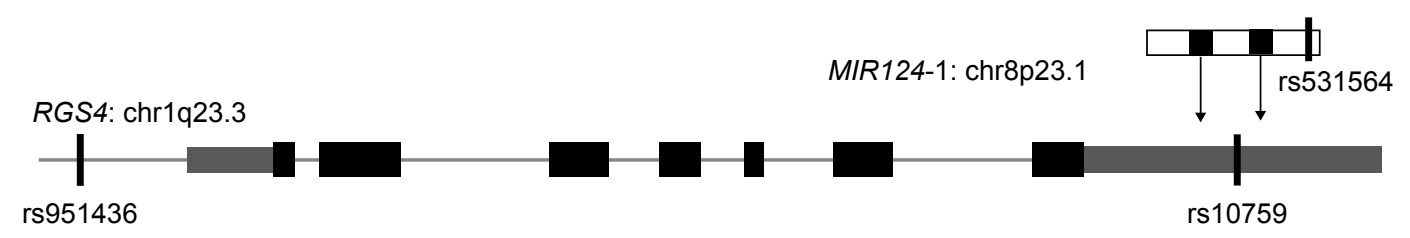

Figure I SNPs in MIRI24-I and its target RGS4 gene. Three polymorphisms: rs53I564 (a functional SNP in MIRI24-I), rs I0759 (a microRNA-binding site SNP in RGS4), and rs95।436 (a promoter SNP in RGS4).

Abbreviation: SNP, single-nucleotide polymorphism. 
18-65 years, and 3) the scores of the 17-item Hamilton Rating Scale for Depression (HAMD-17) $\geq 18$. The exclusion criteria included: 1) additional current DSM-IV Axis-I Disorders, including schizophrenia, bipolar disorder, anxiety disorder, mental retardation, current/past alcohol, or drug abuse-related disorders; 2) severe physical illness that could be etiologically related to the ongoing depressive episode; 3 ) serious suicide risk; and 4) women who are pregnant or lactating.

An additional 436 normal subjects of Han ethnicity were recruited as controls. Subjects who had severe physical illness or a history of any Axis-I psychiatric disorder, or whose first-degree relatives had psychiatric disorders, were not included.

All subjects participating in this study signed written informed consent. The ethics committee of Shanghai Mental Health Center (Shanghai, China) had approved this study.

\section{Treatment and follow-up}

Of the 147 patients initially recruited for treatment, 103 patients completed the 8-week study. Patients were treated with several antidepressant medications, including paroxetine $(\mathrm{N}=24)$, duloxetine $(\mathrm{N}=13)$, agomelatine $(\mathrm{N}=8)$, venlafaxine $(\mathrm{N}=13)$, and bupropion $(\mathrm{N}=45)$. All drugs were used as monotherapy in this study. All patients had a medication washout for a minimum of five half-lives of all current medications. No other medications, including antipsychotics, anxiolytics, or other antidepressants, were allowed in the study, except for medication for insomnia, such as zolpidem $(\mathrm{N}=1)$, zopiclone $(\mathrm{N}=4)$, or clonazepam $(\mathrm{N}=1)$. The daily antidepressant and insomnia drug doses were decided by clinicians based on clinical guidelines and patient responses. The clinical symptoms of the patients were evaluated regularly at baseline (week 0), week 2 , week 4 , week 6, and week 8 with the HAMD-17, the MontgomeryÅsberg Depression Rating Scale (MADRS), and the Clinical Global Impression (CGI) scale. The responders were defined as those with a $50 \%$ reduction in baseline Hamilton score at the end of week 8, while the remitters were defined as having an HAMD-17 score $\leq 7$.

\section{SNP genotyping}

Genomic DNA was extracted from peripheral blood using a Tiangen DNA isolation kit (Blood genomic DNA extraction kit; Tiangen Biotech, Inc., Beijing, China). The three polymorphisms were genotyped using TaqMan assays (Thermo Fisher Scientific, Waltham, MA, USA). Standard polymerase chain reaction (PCR) for amplification was carried out in a $5 \mu \mathrm{L}$ volume, containing $2.50 \mu \mathrm{L}$ of master mix, $2.25 \mu \mathrm{L}$ of DNA, and $0.25 \mu \mathrm{L}$ of TaqMan probe. The PCR amplification was performed with an initial incubation at $95^{\circ} \mathrm{C}$ for 10 minutes, then 40 cycles of $95^{\circ} \mathrm{C}$ for 15 seconds and $60^{\circ} \mathrm{C}$ for 1 minute. After amplification, the allele-specific fluorescence was measured on ABI PRISM ${ }^{\circledR} 7900$ Sequence Detection Systems (Thermo Fisher Scientific). The call rates of genotyping were above $98.9 \%$ for each SNP. For quality control, 10\% of the samples were repeated, and the results were $100 \%$ consistent.

\section{Statistical analysis}

Continuous variables of demographic data were expressed as mean \pm standard deviation and determined by the independent samples $t$-test. Categorical variables of demographic data were presented as frequency in percent (\%) and were analyzed by a chi-squared test. Hardy-Weinberg equilibrium (HWE) and the association analysis of allelic and genotypic frequencies between the MDD and control groups were performed on the SHEsis online software (http://analysis.bio-x. cn/myAnalysis.php). ${ }^{18}$ SNPStats (http://bioinfo.iconcologia. net/snpstats/start.htm) was used for single marker analysis to calculate the odds ratio (OR) of each SNP, using age and gender as covariates. ${ }^{19}$ QUANTO software (version 1.2.4.) (University of Southern California, Los Angeles, CA, USA) was used to calculate the statistic power of the case-control sample size, assuming the prevalence of MDD to be $2 \%{ }^{20}$ and the OR to be 1.5 , as the minor allele frequency of the selected SNPs fluctuated from $17.3 \%$ to $44 \%$ and the power of the sample ranged from $80.82 \%$ to $93.62 \%$. Gene-gene interactions were determined by multifactor dimensionality reduction (MDR) analysis. ${ }^{21}$

Chi-squared test was used to compare the allelic and genotypic distribution of the three polymorphisms between remitters and non-remitters and responders and non-responders. Overall repeated measures analysis of variance was performed to assess the effect of variants on HAMD percent change scores over 8 weeks of antidepressant treatment (genotype as fixed factor, weeks as repeated measures), and age and gender were included as covariates. These statistical analyses were performed using SPSS (version 19; IBM Corporation, Armonk, NY, USA). Results were considered significant with an alpha level $<0.05$ (two-sided test). Bonferroni correction was applied by multiplying the $p$-values by the number of SNPs. With this level of significance, for single marker analysis in our sample, we had a power of 0.80 to detect a medium effect size of $d=0.395$, which corresponded to a difference of approximately $10.02 \%$ HAM-D reduction between two main genotype variants and to an explained variance of $\sim 3.6 \%{ }^{22}$ 


\section{Results}

\section{Demographic and clinical characteristics of subjects}

There was a significant difference in gender distribution between MDD patients ( 82 males and 143 females) and healthy controls ( 152 males and 284 females, $p=0.000$ ). No difference was detected in age between MDD patients and healthy controls ( $36.56 \pm 12.46$ vs $38.38 \pm 11.65, p=0.063$ ).

Of the 147 patients, 103 completed the 8-week follow-up, two withdrew because of adverse events, eight withdrew because of improvement, and 34 for other reasons or were lost to follow-up. The demographic data of patients with MDD who completed 8-week antidepressant treatment are summarized in Table 1. There was no significant difference in gender distribution, duration of current episode, duration of illness, baseline HAMD score, baseline MADRS scores, and baseline CGI scores between responders and non-responders as well as remitters and non-remitters. The mean age for remitters was significantly greater than that for non-remitters ( $\mathrm{t}=2.334, p=0.022$ ), but no significant difference was found between responders and non-responders.

\section{Association of SNPs with the risk of MDD}

The distributions of allele frequencies for the three polymorphisms in patients and controls were in $\operatorname{HWE}(p>0.05)$. There were no significant differences in the distribution of allelic and genotypic frequencies between case and control groups (Table 2).

\section{Analysis of gene-gene interactions}

Gene-gene interactions among three SNPs of MIR124-1 (rs531564) and RGS4 (rs10759, rs951436) genes were analyzed by MDR software, and these three SNPs were brought into the construction of the MDR model (Table 3).
In the optimal model containing rs531564, rs10759, and rs 951436 SNPs, the $p$-value was 0.0024 , the accuracy of the sample test was 0.49 , and the cross-validation consistency was 10/10. Values of ORs and $95 \%$ confidence interval (CI) indicated that the combined action could increase the risk of MDD $(\mathrm{OR}=1.67,95 \% \mathrm{CI}=1.20-2.33)$. As shown in Figure 2A, we found a positive interaction effect of rs 531564 in the MIR124-1 gene and rs10759 in the RGS4 gene with an interaction entropy of $0.14 \%$ and a negative interaction effect of rs 10759 and rs951436 in RGS4 with an interaction entropy of $-0.13 \%$. The interaction dendrogram demonstrated that rs531564 and rs10759 were estimated to have the strongest interaction synergy, as indicated visually by the red line (Figure 2B). All the results indicated that there existed potential gene-gene interactions among MIR124-1 and RGS4 genes, and they might work together in the pathogenesis of MDD.

\section{Association of rs95।436 with response and remission status}

The association of antidepressants with the distribution of allelic and genotypic frequencies for the three polymorphisms (rs951436, rs531564, and rs10759) is shown in Table 4. A significant association was found in genotypic frequencies of rs 951436 between the responder and non-responder groups $\left(\chi^{2}=6.191, p=0.045\right)$ as well as between the remitter and nonremitter groups $\left(\chi^{2}=7.216, p=0.026\right)$, but the significance disappeared after Bonferroni correction (correction $p=0.135$ and $p=0.078$, respectively). For further analysis, the rs 951436 heterozygote carriers had threefold probabilities of achieving clinical complete remission $(\mathrm{OR}=3.00,95 \% \mathrm{CI}=1.33-6.76$, $p=0.007$, correction $p=0.021)$ and 3.21 -fold probabilities of achieving clinical response $(\mathrm{OR}=3.21,95 \% \mathrm{CI}=1.13-9.14$, $p=0.022$, correction $p=0.066$ ) as compared with rs 951436 homozygotes $(\mathrm{AA}+\mathrm{CC})$ after 8-week treatment. In addition,

Table I Demographic and clinical characteristics of subjects

\begin{tabular}{|c|c|c|c|c|c|c|c|c|c|}
\hline Characteristics & $\begin{array}{l}\text { Overall } \\
(\mathrm{N}=103)\end{array}$ & $\begin{array}{l}\text { Remitters } \\
(\mathbf{N}=55)\end{array}$ & $\begin{array}{l}\text { Non-remitters } \\
(\mathrm{N}=\mathbf{4 8})\end{array}$ & $\chi^{2}$ or $\mathbf{t}$ & $p$-value & $\begin{array}{l}\text { Responders } \\
(\mathrm{N}=\mathbf{8 2})\end{array}$ & $\begin{array}{l}\text { Non-responders } \\
(\mathbf{N}=\mathbf{2} \text { I) }\end{array}$ & $\chi^{2}$ or $\mathbf{t}$ & $p$-value \\
\hline \multicolumn{10}{|l|}{ Sex } \\
\hline Male, n (\%) & 48 & $25(52.1)$ & $23(47.9)$ & 0.062 & 0.803 & $36(75.0)$ & $12(25.0)$ & 1.178 & 0.278 \\
\hline Female, n (\%) & 55 & $30(54.5)$ & $25(45.5)$ & & & $46(83.6)$ & $9(16.4)$ & & \\
\hline Age (years) & $36.56 \pm 12.46$ & $37.42 \pm 12.64$ & $32.27 \pm 9.69$ & 2.334 & 0.022 & $35.09 \pm 11.72$ & $34.76 \pm I I .40$ & 0.113 & 0.910 \\
\hline Duration of current & $17.32 \pm 46.94$ & $17.35 \pm 53.36$ & $17.29 \pm 38.54$ & 0.006 & 0.995 & $|8.08 \pm 5| .48$ & $14.05 \pm 17.78$ & 0.318 & 0.751 \\
\hline \multicolumn{10}{|l|}{ episode (months) } \\
\hline Duration of illness (months) & $51.43 \pm 87.39$ & $55.57 \pm 114.65$ & $47.18 \pm 46.38$ & 0.413 & 0.681 & $49.00 \pm 94.51$ & $60.41 \pm 55.07$ & -0.461 & 0.646 \\
\hline Baseline HAMD-I7 scores & $23.57 \pm 2.77$ & $23.20 \pm 2.72$ & $24.00 \pm 2.80$ & -1.468 & 0.145 & $23.46 \pm 2.71$ & $24.00 \pm 3.03$ & -0.739 & 0.466 \\
\hline Baseline MADRS scores & $30.1 I \pm 4.6 I$ & $30.11 \pm 4.59$ & $30.10 \pm 4.68$ & 0.005 & 0.996 & $30.09 \pm 4.65$ & $30.19 \pm 4.59$ & -0.093 & 0.926 \\
\hline Baseline CGI scores & $4.28 \pm 0.60$ & $4.25 \pm 0.62$ & $4.31 \pm 0.60$ & -0.486 & 0.628 & $4.28 \pm 0.61$ & $4.29 \pm 0.56$ & -0.037 & 0.970 \\
\hline
\end{tabular}

Notes: Bold number represents significant $p$-value. Data are presented as mean \pm standard deviation unless otherwise indicated.

Abbreviations: HAMD-17, I7-item Hamilton Rating Scale for Depression; MADRS, Montgomery-Åsberg Depression Rating Scale; CGI, Clinical Global Impression. 
Table 2 Allele and genotype analysis of rs 10759, rs531564, and rs95 I436 between cases and controls

\begin{tabular}{|c|c|c|c|c|c|c|c|c|c|c|c|c|}
\hline SNPs & $\mathbf{N}$ & Allele (\%) & & $x^{2}$ & $p$-value & MAF (\%) & Genotype & (\%) & & $x^{2}$ & $p$-value & OR (95\% Cl) \\
\hline rs10759 & & G & $\mathrm{T}$ & & & & GG & GT & TT & & & \\
\hline Case & 219 & $257(0.587)$ & $181(0.413)$ & 2.811 & 0.094 & 0.44 & $74(0.338)$ & $109(0.498)$ & $36(0.164)$ & 2.983 & 0.225 & $0.78(0.51-1.19)$ \\
\hline Control & 435 & $468(0.538)$ & $402(0.462)$ & & & & $12 \mid(0.278)$ & $226(0.520)$ & $88(0.202)$ & & & \\
\hline rs53I564 & & $\mathrm{C}$ & G & & & & $\mathrm{CC}$ & CG & GG & & & \\
\hline Case & 220 & $76(0.173)$ & $364(0.827)$ & 1.167 & 0.326 & 0.173 & $6(0.027)$ & $64(0.291)$ & I $50(0.682)$ & I. 148 & 0.563 & $1.08(0.39-2.96)$ \\
\hline Control & 435 & $132(0.152)$ & $738(0.848)$ & & & & II (0.025) & $110(0.253)$ & $314(0.722)$ & & & \\
\hline rs95I436 & & A & $\mathrm{C}$ & & & & $\mathrm{AA}$ & $A C$ & $\mathrm{CC}$ & & & \\
\hline Case & 220 & $212(0.482)$ & $228(0.5 \mid 8)$ & 2.379 & 0.123 & 0.41 & $50(0.227)$ & I I (0.509) & $58(0.264)$ & 4.002 & 0.135 & $1.08(0.73-1.60)$ \\
\hline Control & 436 & $38 I(0.437)$ & $49 \mid(0.563)$ & & & & $93(0.213)$ & $195(0.447)$ & 148 (0.339) & & & \\
\hline
\end{tabular}

Abbreviations: MAF, minor allele frequency; OR, odds ratio; $95 \% \mathrm{Cl}, 95 \%$ confidence interval; SNPs, single nucleotide polymorphisms.

there was a trend for G-allele carriers of rs10759 to have lower remission rates than homozygous TT $\left(\chi^{2}=5.098\right.$, $p=0.069$, correction $p=0.207$ ).

No association was found in genotypic frequencies of the other two SNPs. There were no significant differences in the allelic frequencies of the three SNPs between responders and non-responders and remitters and non-remitters ( $p>0.05)$.

Considering that insomnia medications may influence the antidepressant effect, the six patients taking insomnia drugs were removed for more precise analysis. Their removal did not affect the results reported here when comparing the three SNPs with response and remission status (data not shown).

\section{Association of rs95। 436 with HAMD-17 score changes}

Using the repeated measures analysis of covariance for HAM-D percent change scores from admission to week 8, no significant differences were found across the genotypes of the three polymorphisms (rs951436, rs531564, and rs10759) (Figure S1). For further analysis, the homozygous (AA+CC) of rs951436 showed a worse response to antidepressant treatment and had lower percent reduction of HAM-D scores over 8 weeks than heterozygous AC (Figure 3), and significant associations were found at week $6(\mathrm{AA}+\mathrm{CC}$ vs AC: $53.58 \pm 27.04$ vs $61.34 \pm 21.54, \mathrm{t}=-2.08, p=0.040)$ and week 8 (AA+CC vs AC: $60.17 \pm 29.56$ vs $70.19 \pm 20.41, \mathrm{t}=-2.404$, $p=0.018$ ) after the adjustment of age and gender. However, this significance disappeared after Bonferroni correction ( $p_{\mathrm{wk} 6}=0.120$ and $p_{\mathrm{wk} 8}=0.054$, respectively).

Likewise, for more precise analysis, we removed those six samples mentioned above for further analysis. After removing them, no results were significantly altered when comparing three SNPs with HAMD-17 score changes (data not shown).

\section{Discussion}

To our knowledge, this is the first study to investigate the association of polymorphisms of MIR124-1 (rs531564) and its target RGS4 (rs10759 and rs951436) with MDD and antidepressant treatment. miR-124 is a brain-specific miRNA, which is highly expressed in the developing and adult nervous system. ${ }^{4}$ rs531564 as a functional variant in MIR124-1 gene can affect the expression levels of mature miR-124 and thus be involved in neuroplasticity and neurosignaling mechanisms. ${ }^{12}$ Contrary to our expectations, we did not find an association of rs531564 with MDD and antidepressant efficacy in allelic and genotype frequencies. In some previous studies, it has also been reported that rs531564 was not associated with impulsiveness, ${ }^{13}$ mesial temporal lobe epilepsy, or Alzheimer's disease. ${ }^{12,23}$ However, because of the limitation of sample size, false-negative results may occur, especially for the correlation between rs531564 and antidepressant response. Thus, these findings need to be validated in a larger sample.

Table 3 MDR models of gene-gene interactions

\begin{tabular}{lllllll}
\hline Model & $\begin{array}{l}\text { Training } \\
\text { bal. ACC }\end{array}$ & $\begin{array}{l}\text { Testing } \\
\text { bal. ACC }\end{array}$ & $\begin{array}{l}\text { CV } \\
\text { consistency }\end{array}$ & $\chi^{2}$ & p-value & OR (95\% CI) \\
\hline rs951436 & 0.5408 & 0.488 I & $8 / 10$ & 4.1687 & 0.0412 & $1.46(1.01-2.09)$ \\
rs10759, rs531564 & 0.5533 & 0.4769 & $5 / 10$ & 5.3841 & 0.0203 & $1.47(1.06-2.04)$ \\
rs10759, rs531564, rs951436 & 0.5676 & 0.4909 & $10 / 10$ & 9.2385 & 0.0024 & $1.67(1.20-2.33)$ \\
\hline
\end{tabular}

Abbreviations: MDR, multifactor dimensionality reduction; Training bal. ACC, training-balanced accuracy; CV consistency, cross-validation consistency; OR, odds ratio; $95 \% \mathrm{Cl}, 95 \%$ confidence interval. 


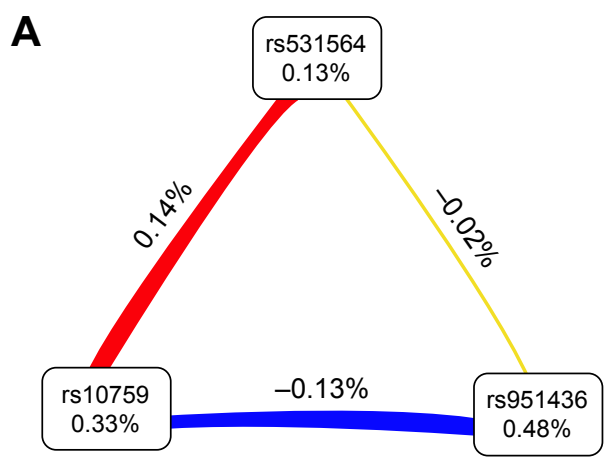

B

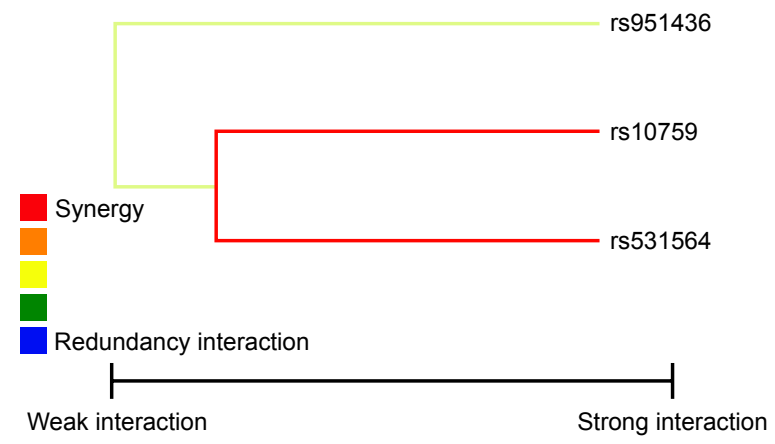

Figure 2 Hierarchical interaction graphs and interaction dendrogram.

Notes: (A) The values inside boxes indicate information gain (IG) of individual attributes or main effects, whereas values between the nodes exemplify IG of pairwise combinations of attributes or interaction effects. A positive entropy (plotted in red or orange) indicates interaction while a negative entropy (plotted in blue) indicates redundancy. (B) Interaction dendrogram shows that the red line represents synergy interaction and the green line represents synergy interaction more weakly. From left to right, the interaction was more intensive.

RGS4 modulates several GPCR signaling cascades that are suggested to have a major role in MDD and antidepressants. ${ }^{24}$ Indeed, increasing evidence suggests that the expression level of RGS4 is altered in patients with MDD and that antidepressant treatments reverse or normalize these effects. ${ }^{15,16}$ Bioinformatics analysis found that rs 951436 was located in the binding sites for transcription factors, such as hepatocyte nuclear factor-6 or lymphoid enhancer binding factor 1/T-cell-specific factor within the putative promoter region of $R G S 4$, which may play a role in transcription regulation. ${ }^{25}$ Increasing evidence has demonstrated that rs951436 is involved in the susceptibility of some mental disorders, such as schizophrenia and bipolar disorder. ${ }^{17,26-28}$ However, in our study, we did not observe a significant association of rs951436 with MDD. To our knowledge, the association of $R G S 4$ polymorphisms with MDD and antidepressants was rarely studied, and only one study showed that rs 951436 was not associated with the risk of treatment-resistant depression in Finland $(\mathrm{n}=119) .{ }^{29}$ Our findings were in accordance with their results.

Although no statistically significant differences in allelic and genotypic frequencies were found between cases and control for the three polymorphisms separately, we found the association between the interaction of MIR124-1 and target gene $R G S 4$ with MDD by using the MDR method. The best gene-gene interaction model identified was a three-locus model (MIR124-1 rs531564, RGS4 rs10759 and rs951436). On the one hand, $R G S 4$ is one of the validated target genes of miR-124, and rs10759, located in the 3' UTRs of RGS4 gene, can alter the binding of miRNA-124 to its target. ${ }^{7}$ Therefore, there may be a functional connection between the two genes. On the other hand, since MDD is a complex

Table 4 The genotype and allele analysis of SNPs between remitters and non-remitters and responders and non-responders

\begin{tabular}{|c|c|c|c|c|c|c|c|c|c|c|}
\hline \multirow[b]{2}{*}{ rs95I436 } & \multicolumn{3}{|c|}{ Genotype $^{a}$} & \multirow[t]{2}{*}{$\chi^{2}$} & \multirow[t]{2}{*}{$p$-value ${ }^{b}$} & \multirow[t]{2}{*}{ Corrected $p$-value ${ }^{c}$} & \multicolumn{2}{|c|}{ Allele } & \multirow[t]{2}{*}{$\chi^{2}$} & \multirow[t]{2}{*}{$p$-value } \\
\hline & AA & $\mathrm{AC}$ & $\mathrm{CC}$ & & & & $A$ & C & & \\
\hline Remitters & 10 & 34 & 10 & 7.216 & 0.026 & 0.078 & 54 & 54 & 0.000 & 1.000 \\
\hline Non-remitters & 15 & 17 & 15 & & & & 47 & 47 & & \\
\hline Responders & 19 & 45 & 16 & 6.191 & 0.045 & 0.135 & 83 & 77 & 1.082 & 0.298 \\
\hline Non-responders & 6 & 6 & 9 & & & & 18 & 24 & & \\
\hline rs53I564 & $\mathrm{CC}$ & CG & GG & & & & C & G & & \\
\hline Remitters & 2 & 13 & 39 & I.342 & 0.511 & NS & 17 & 91 & 0.408 & 0.523 \\
\hline Non-remitters & $\mathrm{I}$ & 16 & 30 & & & & 18 & 76 & & \\
\hline Responders & 3 & 23 & 54 & 1.436 & 0.488 & NS & 29 & $13 \mid$ & 0.342 & 0.558 \\
\hline Non-responders & 0 & 6 & 15 & & & & 6 & 36 & & \\
\hline rs 10759 & GG & GT & TT & & & & $\mathrm{G}$ & $\mathrm{T}$ & & \\
\hline Remitters & 23 & 21 & 10 & 3.711 & 0.156 & NS & 67 & 41 & 0.807 & 0.369 \\
\hline Non-remitters & 20 & 24 & 3 & & & & 64 & 30 & & \\
\hline Responders & 34 & 34 & 12 & 2.035 & 0.361 & NS & 102 & 58 & 0.410 & 0.522 \\
\hline Non-responders & 9 & II & I & & & & 29 & 102 & & \\
\hline
\end{tabular}

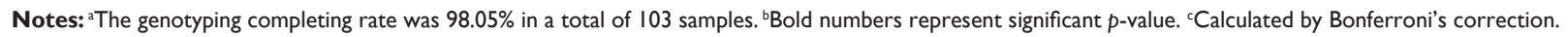
Abbreviation: NS, nonsignificant. 
rs951436

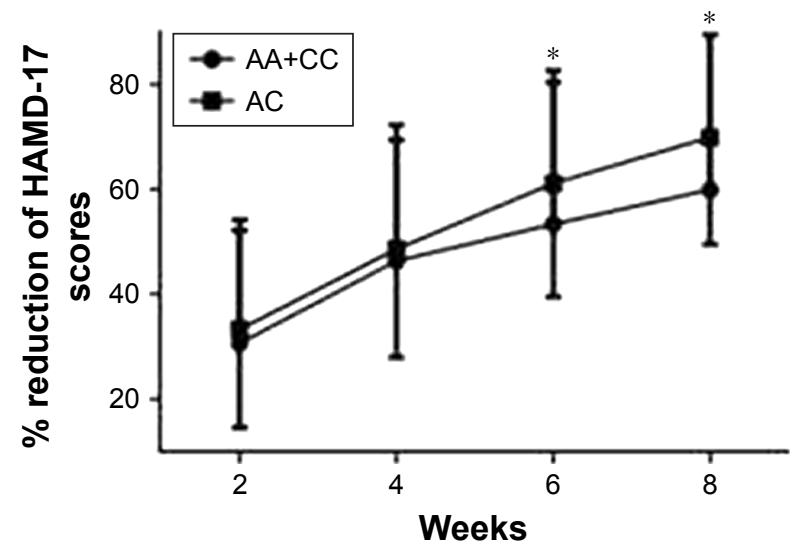

Figure 3 Percent reduction of HAMD-17 scores of antidepressant treatment across genotypes of rs951436.

Notes: Heterozygous (AC) of rs951436 showed a better response to the antidepressant treatment at week 6 and week 8 . The asterisk $\left(^{*}\right)$ represents significant $p$-value.

Abbreviation: HAMD-17, 17-item Hamilton Rating Scale for Depression.

psychiatric disease, we speculate that the individual genetic variants may just display minor marginal effects on its pathogenesis and are difficult to detect, or some of the components, such as MIR124-1 and its target gene RGS4, may act synergistically in the development of MDD in ways we do not understand.

In our study, we found that the heterozygous (AC) of rs951436 may predict a better antidepressant efficacy. On the one hand, rs951436 might have a direct causal effect on response, but the presence of linkage disequilibrium between this SNP and other SNPs in this or other genes that might contribute to this effect is also possible. On the other hand, the reason for this phenomenon might be the positive heterosis effects. Recent studies have demonstrated that low $R G S 4$ expression may cause memory impairment and that overexpression can suppress both behavioral activity and phospho-ERK levels. ${ }^{30,31}$ These findings suggest that both over- and under-expression of RGS4 may be deleterious to normal functioning. Ding et al found that genotypes AA, AC, and $\mathrm{CC}$ of rs951436 were associated with the lowest, moderate, and highest expression levels of $R G S 4$, respectively. ${ }^{25}$ Thus, it seems reasonable to suggest that $R G S 4$ heterozygotes may predict a superior antidepressant response.

We did not find the association of rs 10759 with MDD, but we found a trend for G-allele carriers of rs 10759 to have lower remission rates than homozygous TT. Given the marginal significance, it could mean that the marker makes only a very small contribution to antidepressant response, or it may be due to the relatively small sample. Thus, a larger sample size may be necessary to elucidate a more significant $p$-value.
There are several limitations of our study. First, the sample size was relatively small, especially for the sample size of drug-treated MDD patients. Even so, the statistical analysis showed that the count of the sample size between case and control was sufficient to calculate reliable results. Moreover, the power of the sample for drug-treated MDD patients was sufficient to detect that the effects of variance explained up to $3.6 \%$. Although this is a good result, smaller effects could have been missed. Second, some confounding factors were not considered in the study - including the dosages and categories of the antidepressant and insomnia drugs, first episode or recurrence, and body mass index that may have influenced the response to antidepressant treatment. Third, the SNPs we selected in this study may not provide complete coverage of the MIR124-1 and RGS4 genes. Therefore, our SNP selection method may decrease the power of the study compared with the tag SNP selection method. ${ }^{32}$ We suggest that a variety of MIR124-1 and RGS4 gene polymorphisms are needed in future study to more fully test the association of these two genes and antidepressant treatment response.

In conclusion, our findings implied that an interaction effect of MIR124-1 and RGS4 polymorphisms may play a more important role than individual factors for MDD development. Moreover, this study provides preliminary evidence that RGS4 gene polymorphisms may predict response to antidepressants in MDD in the Chinese population. However, because our samples are small, it will be necessary to replicate and confirm these findings in other independent studies using larger samples.

\section{Acknowledgments}

We gratefully acknowledge the skillful technical support of Mr Dongxiang Wang and Mr Songzhi Zhang. We also thank the patients and healthy volunteers for their participation. This study was supported by grants from the Collaborative Innovation Center for Translational Medicine at Shanghai Jiao Tong University School of Medicine (TM201506, TM201624), National Major Project for IND (2018ZX09734005), and the Programs Foundation of Shanghai Mental Health Center (2017-QH-02).

\section{Disclosure}

The authors report no conflicts of interest in this work.

\section{References}

1. Flynt AS, Lai EC. Biological principles of microRNA-mediated regulation: shared themes amid diversity. Nat Rev Genet. 2008;9(11): $831 \mathrm{e} 842$. 
2. Serafini G, Pompili M, Hansen KF, et al. The involvement of microRNAs in major depression, suicidal behavior, and related disorders: a focus on miR-185 and miR-491-3p. Cell Mol Neurobiol. 2014;34(1):17-30.

3. Bahi A, Chandrasekar V, Dreyer JL. Selective lentiviral-mediated suppression of microRNA124a in the hippocampus evokes antidepressantslike effects in rats. Psychoneuroendocrinology. 2014;46:78-87.

4. Dwivedi Y. microRNA-124: a putative therapeutic target and biomarker for major depression. Expert Opin Ther Targets. 2017;21(7): 653-656.

5. Roy B, Dunbar V, Shelton RC, Dwivedi Y. Identification of microRNA124-3p as a putative epigenetic signature of major depressive disorder. Neuropsychopharmacology. 2017;42:865-875.

6. Cheng LC, Pastrana E, Tavazoie M, Doetsch F. miR-124 regulates adult neurogenesis in the subventricular zone stem cell niche. Nat Neurosci. 2009;12(4):399-408.

7. Gong $\mathrm{Y}, \mathrm{Wu} \mathrm{CN}, \mathrm{Xu} \mathrm{J}$, et al. Polymorphisms in microRNA target sites influence susceptibility to schizophrenia by altering the binding of miRNAs to their targets. Eur Neuropsychopharmacol. 2013;23(10): 1182-1189.

8. Wang SS, Mu RH, Li CF, et al. microRNA-124 targets glucocorticoid receptor and is involved in depression-like behaviors. Prog Neuropsychopharmacol Biol Psychiatry. 2017;79(Pt B):417-425.

9. Liu XS, Chopp M, Zhang RL, et al. MicroRNA profiling in subventricular zone after stroke: miR-124a regulates proliferation of neural progenitor cells through Notch signaling pathway. PLoS One. 2011; 6(8):e23461.

10. Higuchi F, Uchida S, Yamagata H, et al. Hippocampal microRNA-124 enhances chronic stress resilience in mice. J Neurosci. 2016;36(27): 7253-7267.

11. He S, Liu X, Jiang K, et al. Alterations of microRNA-124 expression in peripheral blood mononuclear cells in pre- and post-treatment patients with major depressive disorder. J Psychiatr Res. 2016;78:65-71.

12. Qi L, Hu Y, Zhan Y, Wang J, Wang BB, Xia HF. A SNP site in primiR-124 changes mature miR-124 expression but no contribution to Alzheimer's disease in a Mongolian population. Neurosci Lett. 2012; 515(1):1-6.

13. González-Giraldo Y, Camargo A, López-León S, Adan A, Forero DA. A functional SNP in MIR124-1, a brain expressed miRNA gene, is associated with aggressiveness in a Colombian sample. Eur Psychiatry. 2015;30(4):499-503.

14. Gold SJ, Ni YG, Dohlman HG, Nestler EJ. Regulators of G-protein signaling (RGS) proteins: region-specific expression of nine subtypes in rat brain. J Neurosci. 1997;17(20):8024-8037.

15. Guilloux JP, Douillard-Guilloux G, Kota R, Wang X, Gardier AM, Martinowich K. Molecular evidence for BDNF- and GABA-related dysfunctions in the amygdala of female subjects with major depression. Mol Psychiatry. 2012;17(11):1130-1142.

16. Stratinaki M, Varidaki A, Mitsi V, et al. Regulator of $\mathrm{G}$ protein signaling 4 [corrected] is a crucial modulator of antidepressant drug action in depression and neuropathic pain models. Proc Natl Acad Sci U S A. 2013; 110(20):8254-8259.
17. Buckholtz JW, Meyer-Lindenberg A, Honea RA, et al. Allelic variation in RGS4 impacts functional and structural connectivity in the human brain. J Neurosci. 2007;27(7):1584-1593.

18. Shi YY, He L. SHEsis, a powerful software platform for analyses of linkage disequilibrium, haplotype construction, and genetic association at polymorphism loci. Cell Res. 2005;15(2):97-98.

19. Solé X, Guinó E, Valls J, Iniesta R, Moreno V. SNPStats: a web tool for the analysis of association studies. Bioinformatics. 2006;22(15): 1928-1929.

20. Shen YC, Zhang MY, Huang YQ, et al. Twelve-month prevalence, severity, and unmet need for treatment of mental disorders in metropolitan China. Psychol Med. 2006;36(2):257-267.

21. Ritchie MD, Hahn LW, Roodi N, et al. Multifactor-dimensionality reduction reveals high-order interactions among estrogen-metabolism genes in sporadic breast cancer. Am J Hum Genet. 2001;69:138-147.

22. Cohen J. Statistical Power Analysis for the Behavioral Sciences. Hillsdale, NJ: Lawrence Erlbaum Associates; 1988:8-14.

23. Manna I, Labate A, Borzì G, et al. An SNP site in pri-miR-124, a brain expressed miRNA gene, no contribution to mesial temporal lobe epilepsy in an Italian sample. Neurol Sci. 2016;37(8):1335-1339.

24. Hollinger S, Hepler JR. Cellular regulation of RGS proteins: modulators and integrators of G protein signaling. Pharmacol Rev. 2002; 54(3):527-559.

25. Ding L, Styblo M, Drobná Z, Hegde AN. Expression of the longest RGS4 splice variant in the prefrontal cortex is associated with single nucleotide polymorphisms in schizophrenia patients. Front Psychiatry. 2016;7:26.

26. Chowdari KV, Bamne M, Wood J, Talkowski ME, Mirnics K, Levitt P. Linkage disequilibrium patterns and functional analysis of RGS4 polymorphisms in relation to schizophrenia. Schizophr Bull. 2008;34(1):118-126.

27. de Castro-Catala M, Cristóbal-Narváez P, Kwapil TR, et al. Association between RGS4 variants and psychotic-like experiences in nonclinical individuals. Eur Arch Psychiatry Clin Neurosci. 2017;267(1):19-24.

28. Li Y, Zhao Q, Zhang Z, et al. Association study between RGS4 and bipolar disorder in the Chinese Han population. Psychiatr Genet. 2010; 20(3):130-132.

29. Huuhka K, Kampman O, Anttila S, et al. RGS4 polymorphism and response to electroconvulsive therapy in major depressive disorder. Neurosci Lett. 2008;437(1):25-28.

30. Jung S, Son H, Lee DH, et al. Decreased levels of RGS4 in the paraventricular nucleus facilitate GABAergic inhibition during the acute stress response. Biochem Biophys Res Commun. 2016;472(1):276-280.

31. Schwendt M, Sigmon SA, McGinty JF. RGS4 overexpression in the rat dorsal striatum modulates mGluR5- and amphetamine-mediated behavior and signaling. Psychopharmacology. 2012;221(4):621-635.

32. Stram DO. Tag SNP selection for association studies. Genet Epidemiol. 2004;27(4):365-374. 


\section{Supplementary material}
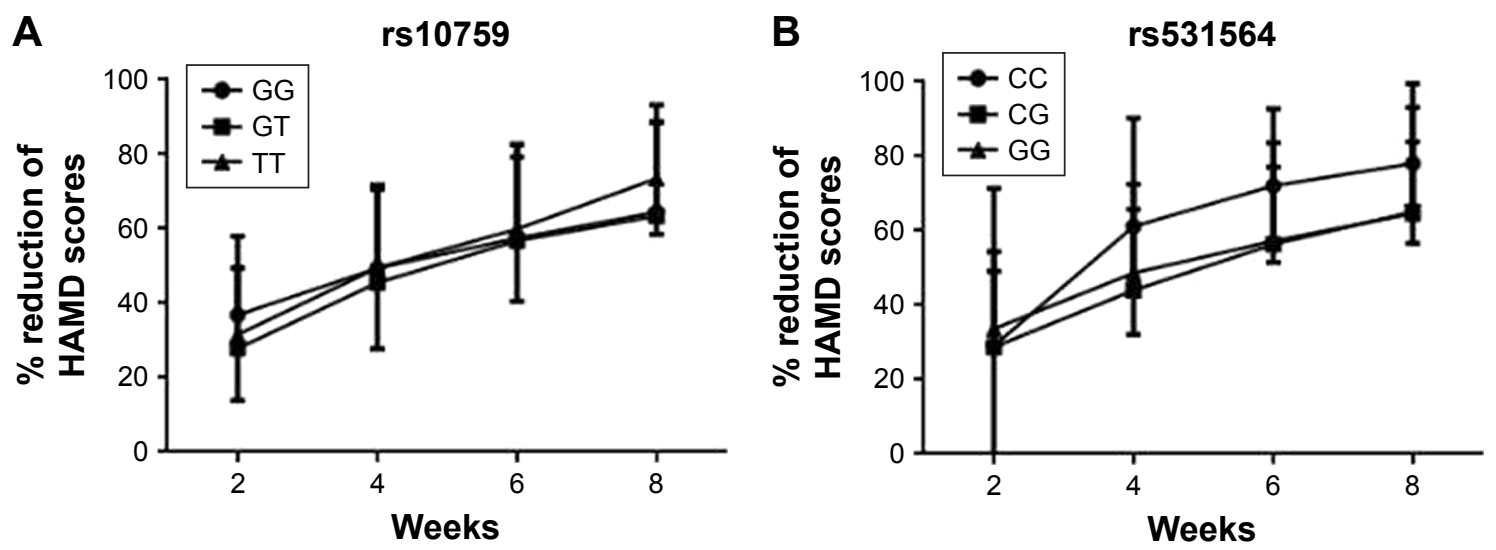

C

rs951436

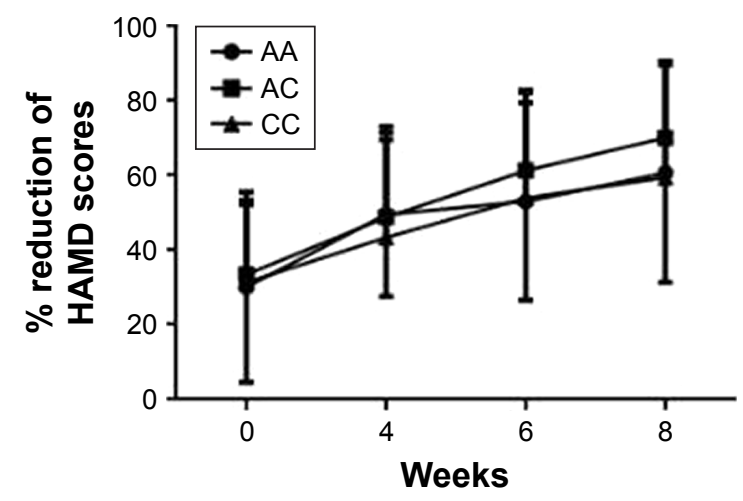

Figure SI Percent reduction of Hamilton Rating Scale for Depression (HAMD) scores of antidepressant treatment across genotypes of rs 10759 (A), rs531563 (B), and rs951436 (C). No significant differences were found between the three polymorphisms and antidepressant.

\section{Publish your work in this journal}

Neuropsychiatric Disease and Treatment is an international, peerreviewed journal of clinical therapeutics and pharmacology focusing on concise rapid reporting of clinical or pre-clinical studies on a range of neuropsychiatric and neurological disorders. This journal is indexed on PubMed Central, the 'PsycINFO' database and CAS, and is the official journal of The International Neuropsychiatric Association (INA). The manuscript management system is completely online and includes a very quick and fair peer-review system, which is all easy to use. Visit http://www.dovepress.com/testimonials.php to read real quotes from published authors. 